\title{
On Quantitative Analysis Model for the Dynamics of the Effect of Parental Background on Pupil/Students Performance in Mathematics
}

\author{
Bassey B. Echeng* \\ Department of Mathematical /Statistics, Cross River University of Technology, 540252, Calabar, Nigeria \\ *Corresponding author: awaserex@ymail.com
}

\begin{abstract}
In this paper, using ordinary differential equations, mathematical performance model was formulated to study the dynamics of pupil/students' performance in mathematics as a function of parental background, incorporating a number of environmental factors. The model considered 8 subgroups, which led to derivation of 8Dimensional dynamic mathematical model for the study of students' performance in mathematics. Model analysis explored numerical methods and the computational simulations of the model indicated that the proportion of pupil/students' from parents with probability of transmission of hereditary and acquired intelligence exhibited high performance in the subject. However, under a cozy environmental factors, male pupil/students' possess more of acquired intelligence in mathematical, whereas, the females exhibited dominance and are sharper via hereditary intelligence. The model therefore, recommended devotion of attention and resources by parents on acquired intelligence of their pupil/students'; as well as both governmental and non-governmental agencies willingness to compliment efforts of parents in the provision of appropriate environment for the enhancement of pupil/students' performance in mathematics. Furthermore, the optimal control and broader predominant studying parameters for similar model is highly encouraged.
\end{abstract}

Keywords: formative-stage, student's-performance, parental-background, hereditary, acquired-intelligence

Cite This Article: Bassey B. Echeng, "On Quantitative Analysis Model for the Dynamics of the Effect of Parental Background on Pupil/Students Performance in Mathematics." Applied Mathematics and Physics, vol. 5, no. 2 (2017): 28-39. doi: 10.12691/amp-5-2-1.

\section{Introduction}

Generally, an in-depth view of the subject mathematics in any dimension cannot be appreciated without a brief mention of modern education. Modern education is known to have been brought to African countries around 1842, notably by the Roman Catholic Missions and Wesleyan Methodist Missionary [1].

In line with several other definitions, education has been defined as an act of training, teaching morally and mentally as well as changing the behavior, feeling, thinking of the learner, see for examples, [2,3,4]. Imbibing the above qualities on any child is a primary function of parents and/or guardians, whose responsibility are to characterize the intelligence of their children while at their formative stage of development (where their brain could take-in easily). This fact was affirmed by [5], who stated that the intelligent of a student can be better predicted from the educational level of his parent. From the above point of view, this paper tends to define education as the training of youngsters between the ages of 6 to 18 years, which spelt the formative stage of any child.

Therefore, mathematics as an offspring of education can be traced as far back as 250 B.C., when the Greek mathematician, Archimedes assumed that the area of a circle was a number; and that the number could be approximated more and more closely by computing the area of regular inscribed polygon of more and more sides $[6,7]$. Mathematics as the name implies, is the science of magnitude and numbers and all their relations [8].

Furthermore, as a result of the vital role of mathematics in all facets of academic endeavor, emphases by teachers, non-governmental and parents in particularly, have been on pupil/students to study the subject (mathematics) effectively. However, pupil/students intelligence in mathematics among other subjects is known to depend on a number of factors. It's obvious that availability of these factors largely depends on parents, and also, the love for their wards and likeness for the subject - mathematics [5].

The model [11] had studied the psychological imprisonment or intellectual freedom? A longitudinal study of contrasting school mathematics approaches and their impact on adults' lives. The study considered 2 schools in England that taught mathematics very differently, the first author found that a project-based mathematics approach resulted in higher achievement, greater understanding, and more appreciation of mathematics than a traditional approach. This follow-up showed that the young adults who had experienced the 2 mathematics teaching approaches developed profoundly 
different relationships with mathematics knowledge that contributed toward the shaping of different identities as learners and users of mathematics.

This present study motivated by the above assertion is design to investigate using mathematical model, as could be found in $[9,10,12]$, the performance of pupil/students in mathematics as a function of their parental background. Thus, the novelty of this present work lies in the explicit inclusion of the study of the hereditary and acquired intelligence of students' as a function of parental background, which make the recent study outstanding compared with the studies of $[1,2,5]$. Moreover, the present model is enhanced 8-Dimensional nonlinear differential equations. Furthermore, the classification of the entire study, which clearly defines the performance of the female students', added a peculiar novelty to this present model.

Other notable mathematical models related to the studies of the effect of students performance in mathematics could be found in $[13,14,15]$.

The entire work is subdivided into five sections, with section 1 , devoted to the introductory aspect. Material and methods are explore in section 2, which define the model parameters and assumptions of the study with which the equation of the model are derived. Derivation of model equations and transformation of equations is performed in section 3. Computer simulations and analysis of result are conducted in section 4 and finally, conclusion and recommendation of the model are inscribed in section 5 .

\section{Material and Methods}

In this section, we define the statement of the problem and formulation of the model equation, guided by a number of assumptions.

\subsection{Statement of the Problem}

In proposing the study for the dynamics of pupil/students' performance in mathematics as a function of parental background, the model explores experimental population consisting of 8 subgroups from which the following model parameters (functions) deduce and define as:

$S_{m}(t)$ - Number of male parents with educational background in mathematics;

$S_{f}(t)$ - Number of female parents with educational background in mathematics;

$I_{m}(t)$ - Number of male pupil/students with hereditary and acquired intelligence from male parents in mathematics;

$I_{m}(t)$ - Number of male pupil/students with hereditary and acquired intelligence from female parents in mathematics;

$W_{m}(t)$ - Number of male pupil/students with only hereditary intelligence in mathematics;

$W_{f}(t)$ - Number of female pupil/students with only hereditary intelligence in mathematics; $U_{m}(t)$ - Number of male pupil/students with only acquired intelligence in mathematics;
$U_{f}(t)$ - Number of female pupil/students with only acquired intelligence in mathematics;

where,

$$
N_{m}(t)=S_{m}(t)+I_{m}(t)+W_{m}(t)+U_{m}(t)
$$

gives the total male population; and

$$
N_{f}(t)=S_{f}(t)+I_{f}(t)+W_{f}(t)+U_{f}(t),
$$

denotes the total female population;

such that

$$
n(t)=N_{m}(t)+N_{f}(t)
$$

Other parameters include:

$R_{m}(t)$ - Rate of male pupil/students with hereditary and acquired intelligence.

$R_{f}(t)$ - Rate of female pupil/students with hereditary and acquired intelligence.

$H_{m}(t)$ - Rate of hereditary intelligence by male pupil/students.

$H_{f}(t)$ - Rate of hereditary intelligence by female pupil/students.

$A_{m}(t)$ - Rate of acquired intelligence by male pupil/students.

$A_{f}(t)$ - Rate of acquired intelligence by female pupil/students.

$b_{m} \quad$ - Recruitment/natural birth rate of male population, $b_{m}>0$;

$b_{f} \quad$ - Recruitment/natural birth rate of male population, $b_{f}>0$;

$\alpha \quad$ - Natural death rate, $\alpha>0$;

$e \quad-$ Conducive environment, $e>0$;

$\mu \quad$ - Availability of textbooks, $\mu>0$;

$q \quad$ - Qualify teachers, $q>0$;

$r \quad$ - Students/Teachers relationship, $r>0$;

$t_{w} \quad$ - Teachers welfare, $t>0$;

$c_{m} \quad$ - Average number of male friends to a female at time $t$;

$c_{f} \quad$ - Average number of female friends to a male at time $t$;

$\beta_{m} \quad$ - Probability of transmission of intelligence by male parents to pupil/students;

$\beta_{f} \quad$ - Probability of transmission of intelligence by male parents to pupil/students;

$\beta_{m}{ }^{*} \quad$ - Probability of acquired intelligence by male pupil/students; and

$\beta_{f}{ }^{*} \quad$ - Probability of acquired intelligence by female pupil/students.

The formulation of the system model further took into account the following limitations:

1. Total population is heterosexual (i.e. consist of male and female);

2. There is vertical transmission of intelligence (i.e. intelligence by hereditary); 
3. Population consist of only pupil/students at their formative stage (i.e. $1 \leq n(t) \leq 18$,years);

4. They exist parents with acquired educational background in mathematics;

5. They exist parents with hereditary educational background in mathematics;

6. Age-structure is ignored; and

7. Emigration and immigration do not occur in the population.

From the system parameters and enlisted limitations, we construct our model schematic flow-chart as seen in Figure 1.

\subsection{Model Formulation}

From Figure 1, and the model parameters as well as the limitations (assumptions) of the model, we drive the model equations as follows:

$$
\begin{gathered}
S_{m}{ }^{\prime}=b_{m} S_{m}+e-R_{m} S_{m} \\
S_{f}^{\prime}=b_{f} S_{f}+e-R_{f} S_{f} \\
I_{m}{ }^{\prime}=R_{m} S_{m}+\left(\mu+q+r+t_{w}\right) I_{m}-\alpha-\left(H_{m}+A_{m}\right) I_{m} \\
I_{f}{ }^{\prime}=R_{f} S_{f}+\left(\mu+q+r+t_{w}\right) I_{f}-\alpha-\left(H_{f}+A_{f}\right) I_{f} \\
W_{m}{ }^{\prime}=H_{m} I_{m}-\alpha+\left(\mu+q+r+t_{w}\right) W_{m} \\
W_{f}^{\prime}=H_{f} I_{f}-\alpha+\left(\mu+q+r+t_{w}\right) W_{f} \\
U_{m}{ }^{\prime}=A_{m} I_{m}-\alpha+\left(\mu+q+r+t_{w}\right) U_{m} \\
U_{f}^{\prime}=A_{f} I_{f}-\alpha+\left(\mu+q+r+t_{w}\right) U_{f}
\end{gathered}
$$

where, $\quad m(t)=\frac{N_{m}(t)}{N_{m}}=S_{m}+I_{m}+W_{m}+U_{m}=1$ and

\section{Equation Transformation and Model Derivation}

In order to reduce the number of equations for explicit analysis of the mathematical implication of both students hereditary and acquired intelligence of mathematics, it becomes obvious to transform our initial models equations (2.1)-(2.8) into proportions i.e.

$$
\begin{aligned}
& x_{m}=\frac{S_{m}}{m(t)} ; x_{f}=\frac{S_{f}}{f(t)} ; y_{m}=\frac{I_{m}}{m(t)} ; y_{f}=\frac{I_{f}}{f(t)} ; \\
& w_{m}=\frac{W_{m}}{m(t)} ; w_{f}=\frac{W_{f}}{f(t)} ; u_{m}=\frac{U_{m}}{m(t)} ; u_{f}=\frac{U_{f}}{f(t)} .
\end{aligned}
$$

Therefore, using the above proportions, the general rate of both hereditary and acquired intelligence for both male and female pupil/students can be defined as follows:

$$
\begin{gathered}
R_{m}(t)=c_{m} \frac{\left(\beta_{m} x_{m}+\beta_{f} x_{f}+\beta_{m}{ }^{*} y_{m}\right)}{m(t)} \\
R_{f}(t)=c_{f} \frac{\left(\beta_{f} x_{f}+\beta_{m} x_{m}+\beta_{f}{ }^{*} y_{f}\right)}{f(t)} \\
H_{m}(t)=c_{m} \frac{\left(\beta_{m} x_{m}+\beta_{f} x_{f}\right)}{m(t)} \\
H_{f}(t)=c_{f} \frac{\left(\beta_{f} x_{f}+\beta_{m} x_{m}\right)}{f(t)} \\
A_{m}(t)=c_{m} \frac{\left(\beta_{m}^{*} y_{m}+\beta_{f}^{*} y_{f}\right)}{m(t)} \\
A_{f}(t)=c_{f} \frac{\left(\beta_{f}^{*} y_{f}+\beta_{m}^{*} y_{m}\right)}{f(t)} .
\end{gathered}
$$

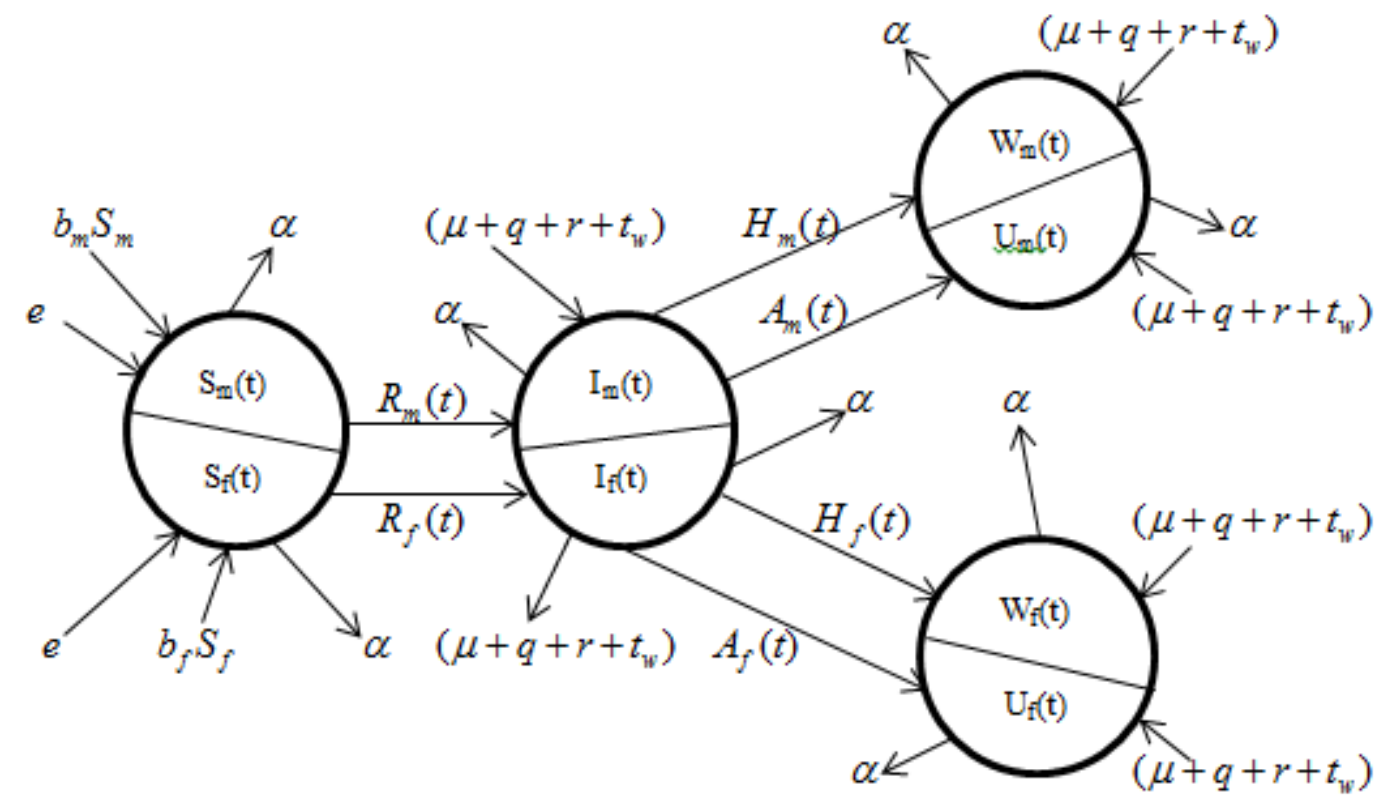

Figure 1. Schematic flow-chart for the effect of parental background in pupil/students' performance in mathematics 
Thus, further application of above derived proportions and substitution of equations (2.9)-(2.14) into equations (2.1)-(2.8), leads to the derivation of desired equations of the system as seen below:

$$
\left.\begin{array}{c}
x_{m}{ }^{\prime}=b_{m} x_{m}+e-\alpha-c_{m} \frac{\left(\beta_{m} x_{m}+\beta_{f} x_{f}+\beta_{m}{ }^{*} y_{m}\right)}{m(t)} x_{m} \\
x_{f}{ }^{\prime}=b_{f} x_{f}+e-\alpha-c_{f} \frac{\left(\beta_{f} x_{f}+\beta_{m} x_{m}+\beta_{f}{ }^{*} y_{f}\right)}{f(t)} x_{f} \\
y_{m}{ }^{\prime}=c_{m} \frac{\left(\beta_{m} x_{m}+\beta_{f} x_{f}+\beta_{m}{ }^{*} y_{m}\right)}{m(t)} x_{m} \\
+\left(\mu+q+r+t_{w}\right) x_{m}-\alpha \\
-\frac{1}{m(t)}\left[\begin{array}{c}
c_{m}\left(\beta_{m} x_{m}+\beta_{f} x_{f}\right) \\
+c_{m}\left(\beta_{m}{ }^{*} y_{m}+\beta_{f}{ }^{*} y_{f}\right)
\end{array}\right] \\
y_{f}^{\prime}=c_{f} \frac{\left(\beta_{f} x_{f}+\beta_{m} x_{m}+\beta_{f}^{*} y_{f}\right)}{f(t)} x_{f} \\
+\left(\mu+q+r+t_{w}\right) x_{f}-\alpha \\
-\frac{1}{f(t)}\left[\begin{array}{c}
c_{f}\left(\beta_{f} x_{f}+\beta_{m} x_{m}\right) \\
+c_{f}\left(\beta_{f}{ }^{*} y_{f}+\beta_{m}^{*} y_{m}\right)
\end{array}\right] \\
w_{m}{ }^{\prime}=c_{m}\left(\frac{\beta_{m} x_{m}+\beta_{f} x_{f}}{m(t)}\right) y_{m}
\end{array}\right\}
$$

\begin{tabular}{|c|c|c|c|c|c|c|c|c|c|c|c|c|c|c|}
\hline variant & $b_{m}$ & $b_{f}$ & $\alpha$ & $e$ & $\mu$ & $q$ & $r$ & $t_{w}$ & $c_{m}$ & $c_{f}$ & $\beta_{m}$ & $\beta_{f}$ & $\beta_{m}{ }^{*}$ & $\beta_{f}{ }^{*}$ \\
\hline 1. & 0.2 & 0.1 & 0.04 & 0 & 0 & 0 & 0 & 0 & 5 & 5 & 0.4 & 0.4 & 0.05 & 0.05 \\
\hline 2. & 0.2 & 0.1 & 0.04 & 0 & 0.5 & 0.5 & $\mathbf{0 . 4}$ & 0.5 & 5 & 5 & 0.4 & 0.4 & 0.05 & 0.05 \\
\hline 3. & 0.2 & 0.1 & 0.04 & 0.5 & 0.5 & 0.5 & $\mathbf{0 . 4}$ & 0.5 & 5 & 5 & 0.4 & 0.4 & $\mathbf{0 . 2}$ & $\mathbf{0 . 5}$ \\
\hline
\end{tabular}

with initial values of all characteristics remaining constant as in Table 2 below:

Table 2. Values for the model variables

\begin{tabular}{|c|c|c|c|c|c|c|c|c|}
\hline Variables & $x_{m}$ & $x_{f}$ & $y_{m}$ & $y_{f}$ & $w_{m}$ & $w_{f}$ & $u_{m}$ & $u_{f}$ \\
\hline Values & 0.2 & 0.1 & 0.2 & 0.1 & 0.1 & 0.1 & 0.15 & 0.05 \\
\hline
\end{tabular}

The numerical experiments are meant to study the dynamics of the effect of parental background on the performance of pupil/students' in mathematics particularly at their formative stage. Furthermore, it is worth to note that in simulating our model for the investigation of students intellectual performance in mathematics, we took into account some parameters, which were considered as predominantly the decisive functions of the parental background. These includes: conducive learning environment, $e$; availability of textbooks, $\mu$; qualify teachers, $q$; students/teachers relationship, $r$; teachers welfare, $t_{w}$.

$$
\begin{aligned}
w_{f}^{\prime}= & c_{f}\left(\frac{\beta_{f} x_{f}+\beta_{m} x_{m}}{f(t)}\right) y_{f} \\
& -\alpha+\left(\mu+q+r+t_{w}\right) w_{f} \\
u_{m}{ }^{\prime}= & c_{m}\left(\frac{\beta_{m}{ }^{*} x_{m}+\beta_{f}{ }^{*} x_{f}}{m(t)}\right) y_{m} \\
& -\alpha+\left(\mu+q+r+t_{w}\right) u_{m} \\
u_{f^{\prime}}= & c_{f}\left(\frac{\beta_{f}{ }^{*} x_{f}+\beta_{m}{ }^{*} x_{m}}{f(t)}\right) y_{f} \\
& -\alpha+\left(\mu+q+r+t_{w}\right) u_{f} .
\end{aligned}
$$

Thus, equations (2.15)-(2.22) define the equations of the model. We next illustrate the derived model.

\section{Numerical Simulations and Discussion}

We denote this section to the numerical illustrations and analysis of our derived model equations, followed by the analysis and discussion of the resulting outcome of our investigation.

\subsection{Numerical Illustrations and Analysis}

In simulating the derived model, a number of illustrative examples are established. The simulations explore the classical in-built Runge-Kutter of order of precision 4 in a Mathcad environment, using a set of deduced established numerical data with varying source $[1,2,5]$ and set of model variables as seen in Table $1 \&$ Table 2 , below: 


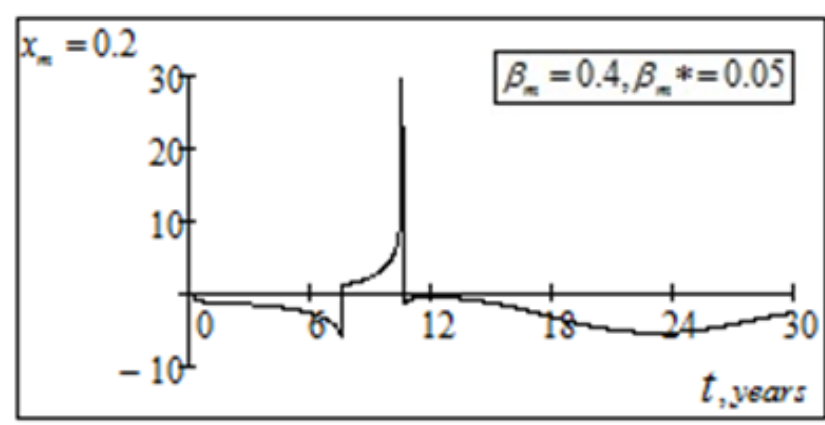

a). Graphical simulation of $x_{m}$ with zero predominant parame ters, $e, \mu, q, r, t_{\mathrm{w}}=0$.

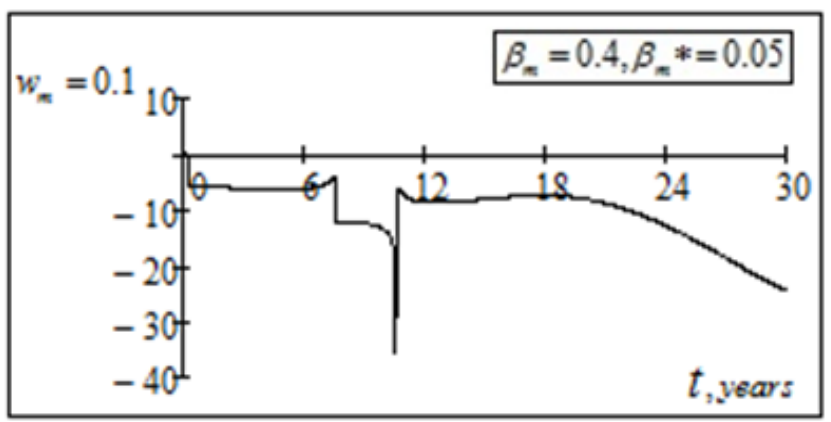

c). Graphical simulation of $w_{*}$ with zero predominant parameters, $e, \mu, q, r, t_{\mathrm{w}}=0$.

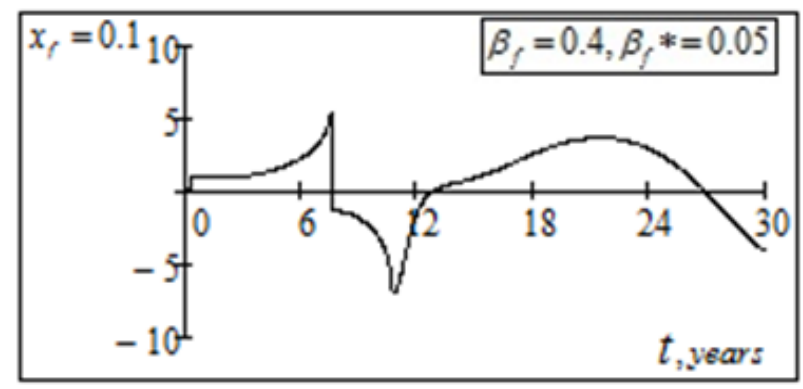

e). Graphical simulation of $x$, with zero predominant parameters, $e, \mu, q, r, t_{\mathrm{w}}=0$.

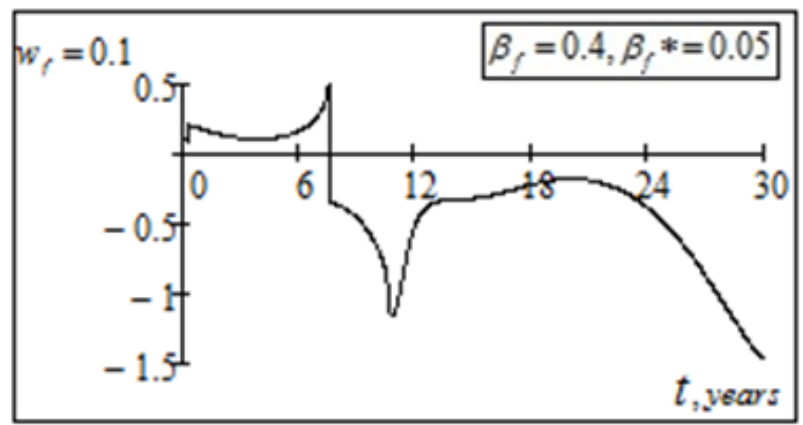

g). Graphical simulation of $w$, with zero predominant parameters, $e, \mu, q, r, t_{\mathrm{w}}=0$.

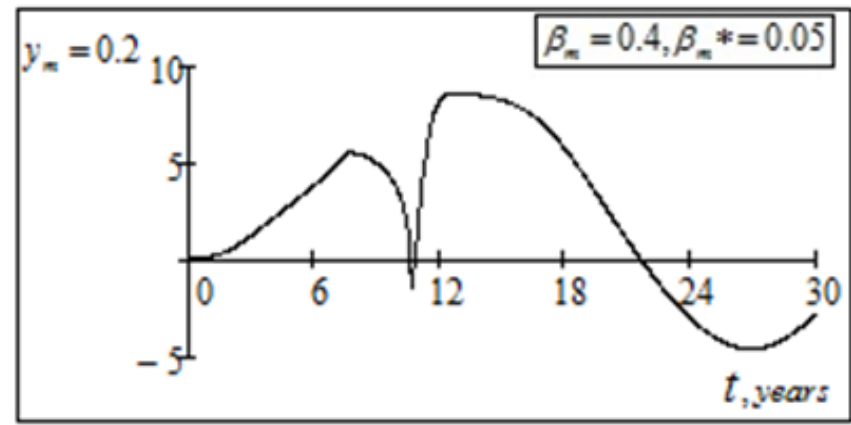

b). Graphical simulation of $y_{n}$ with zero predominant parameters, $e, \mu, q, r, t_{\mathrm{w}}=0$.

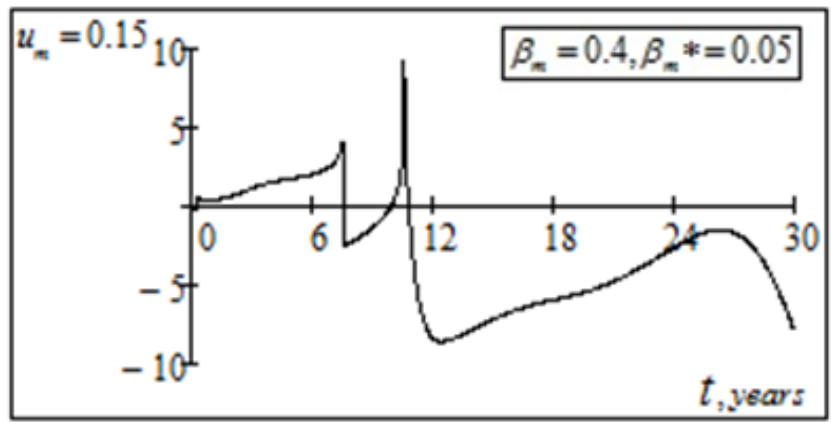

d). Graphical simulation of $u_{n}$ with zero predominant parameters, $e, \mu, q, r, t_{\mathrm{\alpha}}=0$.

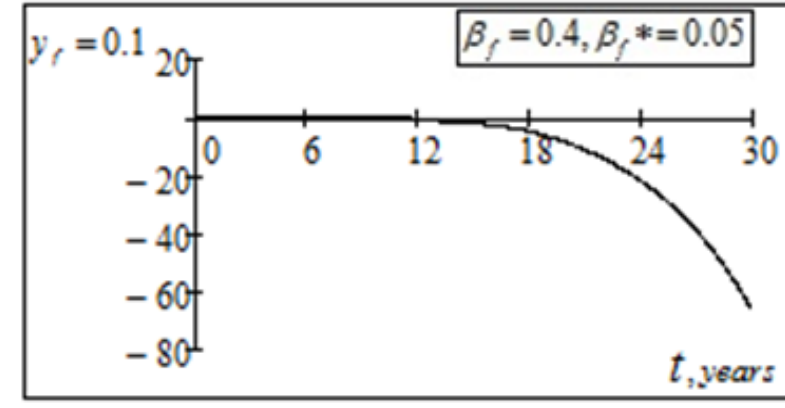

f). Graphical simulation of $y$, with zero predominant parameters, $e, \mu, q, r, t_{\mathrm{w}}=0$.

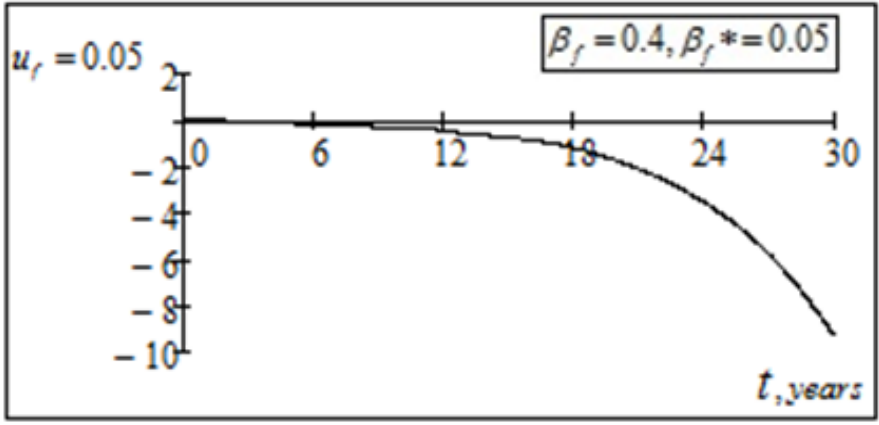

h). Graphical simulation of $u$, with zero predominant parameters, $e, \mu, q, r, t_{\mathrm{w}}=0$.

Figure 2 (a-h). Graphical simulation of male and female pupil/students' performance in mathematics with zero predominant parameters, $e, \mu, q, r, t_{w}=0$

In particular, from Figure 2(a) above, with predominant parameters equal to zero and having other parameters as in variant 1 , of Table 1 , we observe that for a proportion of male parents $x_{m}=0.2$, mathematical intelligence diminishes for a larger part of the investigation, exhibiting sharp interval flash of knowledge of mathematics only at 7-10 years with apex knowledge value of $29.42 \%$.

From Figure 2(b) above, with $y_{m}=0.2$, as the proportion of male pupil/students, and with similar conditions of complete lack of access to conducive 
learning factors i.e. $e, \mu, q, r, t_{w}=0$, the natural combination of hereditary and acquired intelligence of students in mathematics oscillate positively at two different intervals with initial appreciable growth of $5.62 \%$ at the formative stage of 1-7 years. Mathematical intelligence decline at the $10^{\text {th }}$ year and then exhibit expanded knowledge of mathematics reaching apex value of $8.65 \%$ at the $12^{\text {th }}$ year and set a gradual declination through 22 years. At the $27^{\text {th }}$ year, knowledge of mathematics declined to a negative value of $-4.56 \%$.

In Figure 2(c) above, observing similar conditions as in Figure 2(b), we simulate with $w_{m}=0.1$ proportion of male pupil/students, the hereditary behavioral intelligence in mathematics. Result shows complete declination of hereditary intelligence with worse knowledge of mathematics of $-35.74 \%$ at the $10^{\text {th }}$ year. Thus, male hereditary knowledge of mathematics does not exist in the absence of predominant learning factors.

Simulation of male pupil/students with only acquired knowledge of mathematics is study by Figure 2(d) above. For a population proportion of $u_{m}=0.1$, with zero predominant parameters, investigation show that intermittent knowledge of mathematics is seen at the formative stage through 6 years with initial inclination of $4.83 \%$ and then decline thereafter. Intelligence here exhibits a peak flash at the $10^{\text {th }}$ year to $9.27 \%$ and thereafter, decline throughout the period of investigation. Acquired knowledge in mathematics is as weak as $-8.65 \%$ at the $12^{\text {th }}$ year.

Considering the female behavioral performance in mathematics, we simulate for female population proportion $x_{f}=0.1$, female parental background in mathematics. Figure 2(e) above clearly illustrate the fact the under zero predominant parameters, oscillation of mathematical intelligence is observe, which exhibit positive inclination at the early years of study with initial apex of $5.35 \%$ at the $7^{\text {th }}$ year. Intelligence diminishes thereafter through the $10^{\text {th }}$ year with value as low as $-6.79 \%$. Result further indicates gradual appreciation of mathematical intelligence after 12 years with apex value of $4.75 \%$ and finally deteriorated after 26 years of studies.

From Figure 2(f) above, we investigate the intellectual behavior of female pupil/students in mathematics for a combination of hereditary and acquired intelligence. Using a female population of $y_{f}=0.1$ and simulated under zero predominant parameters, result shows insignificant static knowledge of mathematics with marginal brightness of $0.14 \%$ at the formative age through 12 years of studies and the deteriorated through the rest of investigation period.

Figure 2(g) above simulated the situation where $w_{f}=0.1$ proportion of female population was evaluated for hereditary intelligence in mathematics. Under similar conditions as in Figure 2(f), result show that female pupil/students exhibit spatial hereditary knowledge of mathematics at the formative stage i.e. $w_{f}=0.49 \%$ through $7^{\text {th }}$ year. Further observation reveals sharp diminishment of mathematical intelligence after the $7^{\text {th }}$ year and through the duration of experimental investigation.

Finally, for some zero predominant parameters, we conduct as in Figure 2(h) above, the female performance for their level of intelligence of acquired knowledge in mathematics. Result shows a near zero knowledge of mathematics particularly at the formative stage. Gradual extinction of mathematical knowledge is seen after 6 years and through the experimental period. The implication here is that female acquired intelligence in mathematics exists spatially at the formative stage and ceases to exist after the $10^{\text {th }}$ year.

Next, while we allow other parameter values of variant 1 , we intensify our experiment with the activation of some predominant parameters (i.e. $r=0.4 ; \mu, q, \mathrm{t}_{w}=0.5$ ), while $e=0$, as seen in variant 2 , of Table 1 . Maintaining the values of the variables of Table 2, we investigate from Figures 3(a-h) below, the varying intellectual behavior of pupil/students in mathematics.

Figure 3(a) below represents the simulation of male parents' background in mathematics taking into account the conditions given above. Result indicates initial sharp appreciation of parental knowledge of mathematics to a value of $2.11 \%$ and then diminishes gradually through the early 6 years. Parents' knowledge of mathematics gained momentum after 6 years through 12 years exhibiting high tendency of mathematical intelligence at $18-21$ years. Loss of concentration is seen at the $23^{\text {rd }}$ year, which then spurs up with a peak value $5.51 \%$ at the $27^{\text {th }}$ year before exhibiting further undulating knowledge of the subject.

Observing similar conditions of Figure 3(a), we simulate from Figure 3(b) below, for male population proportion of $y_{m}=0.2$, the intelligence performance for a combined hereditary and acquired knowledge of mathematics. Result shows oscillations of male hereditary and acquired intelligence with initial deterioration of mathematical knowledge at the formative stage at $11^{\text {th }}$ year. Brightness in mathematical knowledge oscillates to a value of $y_{m}=48.71 \%$ through $18^{\text {th }}$ year.

From Figure 3(c) below, with similar environmental studying factors as in Figure 3(b) below, we conducted an investigation for only hereditary intellectual behavior of male pupil/students in mathematics. Result vilifies slight knowledge of mathematics at formative stage from 1-7 years and then experience deteriorating knowledge of mathematics within the age range of 8-21 years. Rejuvenation of mathematical intelligence is observe again after 21 years with a peak value of $461.89 \%$ at $27^{\text {th }}$ year and remain stationary thereafter.

Subjection of Figure 3(d) to similar structural learning factors as in Figure 3(c) below, shows that for a male population of $u_{m}=0.15$, the acquired intelligence of this class of pupil/students in mathematics is low at the formative stage through the $11^{\text {th }}$ year. Gradual spanning of mathematical intelligence is seen from the $12^{\text {th }}$ year with mix oscillated cluster intelligence of peak value $4.759 \times 10^{5}$ at the $27^{\text {th }}$ year. Also, we observe a slight deterioration of intelligence at the 26 the year to a value of $-3.51 \times 10^{4}$. 


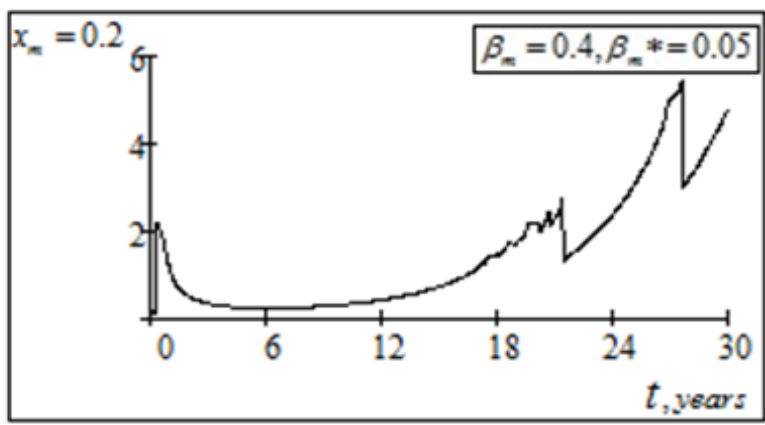

a). Graphical simulation of $x_{m}$ with predominant parameters, $e=0, r=0.4, \mu, q, t_{\mathrm{w}}=0.5$.

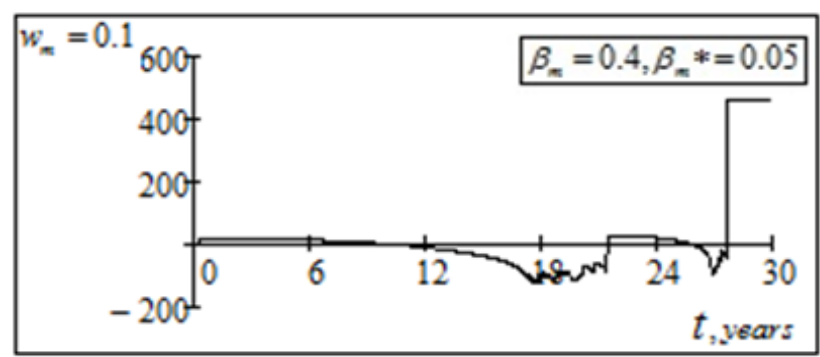

c). Graphical simulation of $w$, with predominant parameters, $e=0, r=0.4, \mu, q, t_{\mathrm{x}}=0.5$.

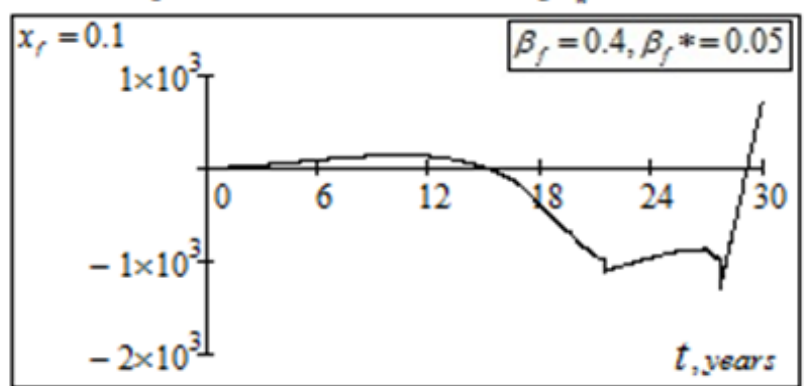

e). Graphical simulation of $x$, with predominant parameters, $e=0, r=0.4, \mu, q, t_{\mathrm{w}}=0.5$.

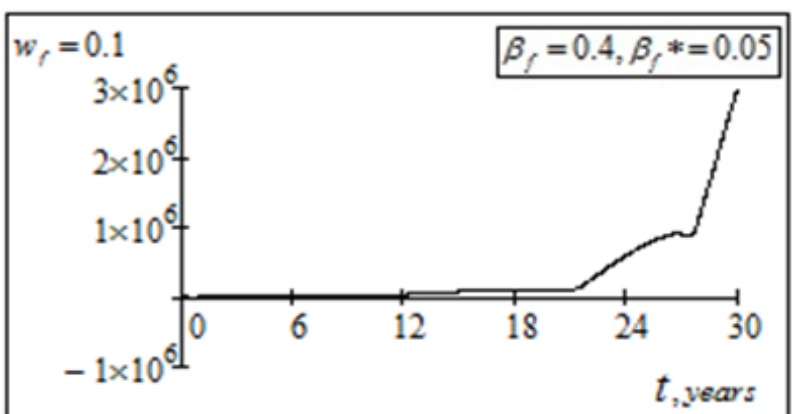

g). Graphical simulation of $w$, with predominant parameters, $e=0, r=0.4, \mu, q, t_{\mathrm{w}}=0.5$.

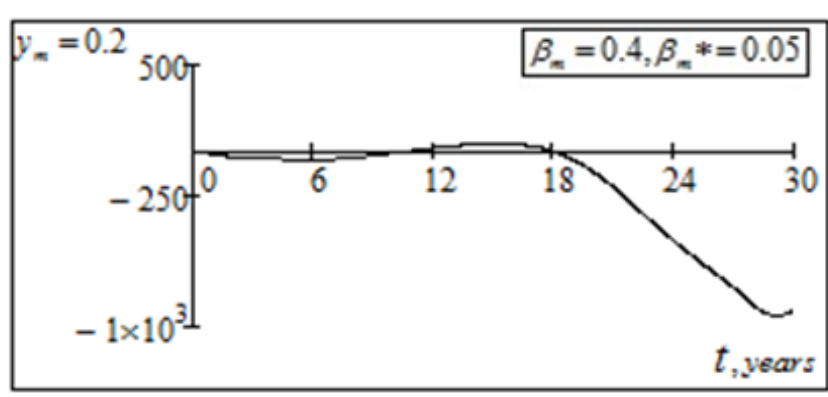

b). Graphical simulation of $y_{n}$ with predominant parameters, $e=0, r=0.4, \mu, q, t_{\mathrm{w}}=0.5$.

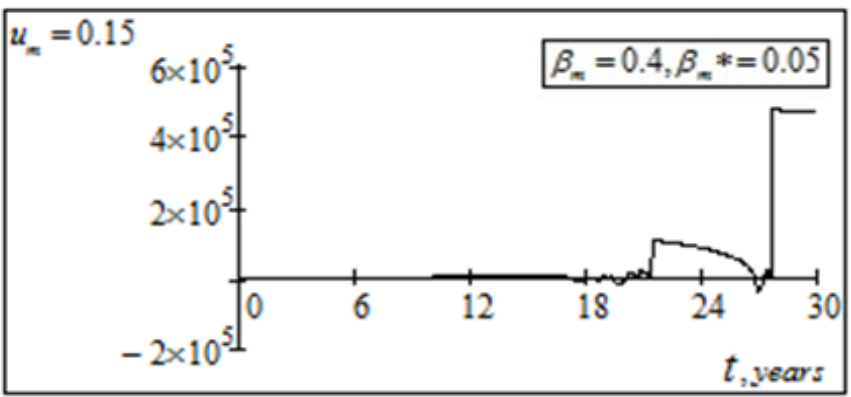

d). Graphical simulation of $u_{m}$ with predominant parameters, $e=0, r=0.4, \mu, q, t_{\mathrm{w}}=0.5$.

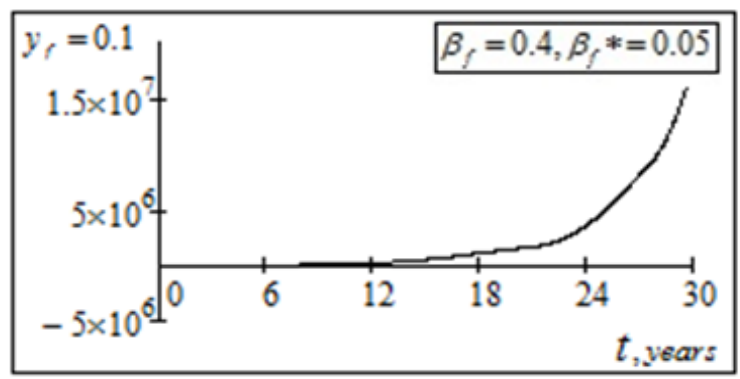

f). Graphical simulation of $y$, with predominant parameters, $e=0, r=0.4, \mu, q, t_{\mathrm{w}}=0.5$.

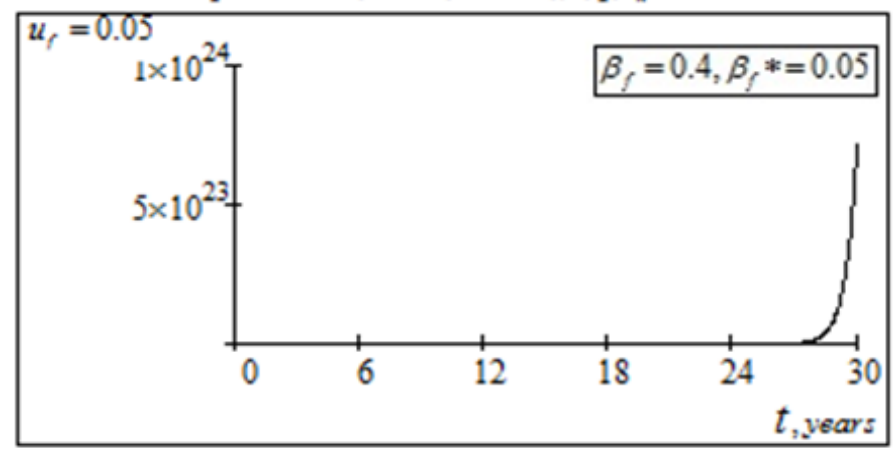

h). Graphical simulation of $u$, with predominant parameters, $e=0, r=0.4, \mu, q, t_{\mathrm{w}}=0.5$.

Figure 3 (a-h). Graphical simulation of male and female pupil/students' performance in mathematics with predominant parameters, $e=0, \mathrm{r}=0.4$, $\mu, q, t_{w}=0.5$

Figure 3(e) above, demonstrates the situation for population of $x_{f}=0.1$ female parental capability in mathematics under predominant environmental parameters as in $x_{m}$, (i.e. $r=0.4 ; \mu, q, \mathrm{t}_{w}=0.5$ ), while $e=0$. Result shows a clear indication of gradual inclination of mathematical knowledge from the formative stage through 16 years, with intelligence level of $0.27 \%$. Mathematical intelligence diminishes from the $17^{\text {th }}$ year with lowest knowledge value of $-1.28 \times 10^{3} \%$ at the $27^{\text {th }}$ year. Resurface of intelligence is seen after the $27^{\text {th }}$ year having climax at $0.75 \times 10^{3} \%$.

Simulation of female pupil/students with a combination of both hereditary and acquired intelligence in mathematics is as represented by Figure 3(f) above. For a 
population proportion of $y_{f}=0.1$ and under similar studying conditions as in Figure 3(e), knowledge of mathematics is docile at formative stage until 12 years and then appreciated gradually from 12 years of studies. Significant brightness of female intelligence in mathematics is visible from 18 years through the rest duration of the investigation with peak level of $1.78 \times 10^{7} \%$.

Figure $3(\mathrm{~g})$ above illustrates female pupil/students with only hereditary intelligence in mathematics for a similar educational environment as in Figure 3(e). The result from $w_{f}=0.1$ population proportion shows low appreciation of mathematics at the formative stage and spanning through 12 years. We observe gradual appreciation of mathematical intelligence from 14 years through 22 years with initial level of $0.67 \times 10^{6}$. Intelligent after 22 years is seen to exhibit some trapezoid inclination and having peak value of $2.99 \times 10^{6}$, which span through 17-30 years of practices in mathematics.

Finally, from Figure 3(h) above, sustaining similar studying environment as in Figures 3(a-g) above, we investigate female pupil/students acquired intelligence in mathematics using population proportion of $u_{f}=0.05$.

Result indicates predominant stable low appreciation of mathematics for a greater lifespan of the students visibly from their formative stage through 26 years. Female acquired intelligence is seen to tremendously spring with apex value of $7.21 \times 10^{23} \%$ at age range of $27-30$ years.

Further intensification of investigation leads to the enhancement of predominant parameters of variant 2, Table 1 above, with inclusive of conducive environment i.e. $e=0.5$. That is, variant 3 of Table 1 , completely represent the enhanced model parameter data and as well, accounted for slight variations on the probability of hereditary and acquired transmission of mathematical intelligence i.e. $\beta_{m}, \beta_{f}=0.4 ; \beta_{m} *=0.2$ and $\beta_{f} *=0.5$, for all the third phase of experiment. The predominant parameters read thus: $r=0.4, e, \mu, q, t_{w}=0.5$.

Observing the above parameter values, we simulate as in Figure 4(a) below, the behavioral intelligence of male parent in mathematics taking a population proportion of $x_{m}=0.2$. Result shows gradual appreciation of mathematical knowledge by male parents after 6 years of study. Onward positive oscillation of mathematical intelligence is seen from the $12^{\text {th }}$ through $27^{\text {th }}$ year with inclinatory peak value of $492.15 \%$ at the $30^{\text {th }}$ year.

For a population of $y_{m}=0.2$, we study as in Figure 4(b) below, the intelligence performance of male pupil/students with both hereditary and acquired knowledge in mathematics. Here, result shows that mathematical intelligence is quite low and tends to remain static along the formative stage through 12 years and then deteriorates through 21 years. Knowledge of mathematics resurfaces from the $22^{\text {nd }}$ year and tremendously appreciated with apex value of $8.44 \times 10^{3} \%$ at $24-30$ years.

Observing similar conditions of Figure 4(b), we simulate as in Figure 4(c) below, and using male population proportion of $w_{m}=0.1$, the hereditary intelligence of male pupil/students in mathematics. Result indicates basic acquisition of mathematical knowledge via hereditary intelligence at the formative stage through 12 years and showing a drop of intelligence after $12^{\text {th }}$ through $14^{\text {th }}$ year. Resurface of hereditary intelligence of male pupil/students in mathematics exhibits spontaneous growth from $15^{\text {th }}$ year through the $30^{\text {th }}$ year of investigation with apex value of $4.15 \times 10^{3} \%$ intelligence level.

Looking at the level of acquired intelligence for male pupil/students in mathematics, we observe from a population proportion of $w_{m}=0.15$ and under similar learning environment of Figure 4(c) that from Figure 4(d) below, a situation posed by low level of acquired intelligence particularly at the formative stage through 10 years and then diminishes to near zero in the interval $10^{\text {th }}$. $14^{\text {th }}$ year. Unsteady dimension of acquired intelligence is seen through the period $15^{\text {th }}-27^{\text {th }}$ years with a spontaneous $8.63 \times 10^{3} \%$ brightness of acquired mathematical intelligence after $27^{\text {th }}$ year through 30 years of the investigation.

On the other hand, we observe the behavioral changes of the female population in mathematics, given similar conditions as their male counterparts. From Figure 4(e) below, we simulate as in variant 3 of Table 1 , the intellectual background of female parents in mathematics. Result show that for a population proportion of $x_{f}=0.1$, the general mathematical intelligence is low at their initial stage through 12 years of study. This is followed by slight deterioration of mathematical intelligence after 12 years through 18 years. Female parental knowledge of mathematics is visible after 18 years through 30 years with inclinative value of $2.82 \times 10^{4} \%$.

Maintaining similar predominant studying environment for the rest of the varying intelligence quota for the female population, we investigate as in Figure 4(f) below, the hereditary and acquired intelligence of female students' performance in mathematics taken a population sample of $y=0.1$. Result shows stationary growth in knowledge for mathematics through 13 years before set of deterioration down to as low as $-4.59 \times 10^{6}$ seen from 14-27 years. Knowledge of mathematics is seen to appreciate thereafter to an apex value $6.18 \times 10^{6}$ from 27 through 30 years.

The hereditary intelligence of female population in mathematics is further investigated as presented by Figure 4(g) below. Result shows lateral growth of female hereditary intelligence in mathematics from the formative stage through 14 years of study with value $w_{f} \leq 1 \times 10^{6}$. The interval $16 \leq w_{f} \leq 27$ years explain the oscillatory diminishment of female hereditary knowledge in mathematics with depreciated apex value of $-5.09 \times 10^{5}$. Incline hereditary knowledge of female intelligence in mathematics is observed at the later period of $w_{f} \geq 27$ years with apex value of $w_{f}=4.03 \times 10^{6}$.

Finally, Figure 4(h) below, investigate the behavioral intelligence of female pupil/students in mathematics under 
the auspices of only acquired intelligence. With population proportion of $u_{f}=0.05$ and under enhanced environmental factors, the female exhibits appreciable brightness in mathematics most specifically at the

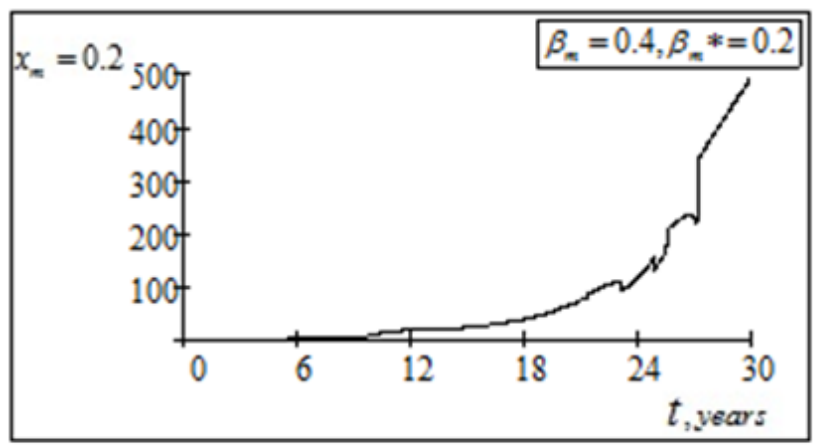

a). Graphical simulation of $x_{m}$ with predominant parameters, $\mathrm{r}=0.4, e, \mu, q, t_{\mathrm{w}}=0.5$

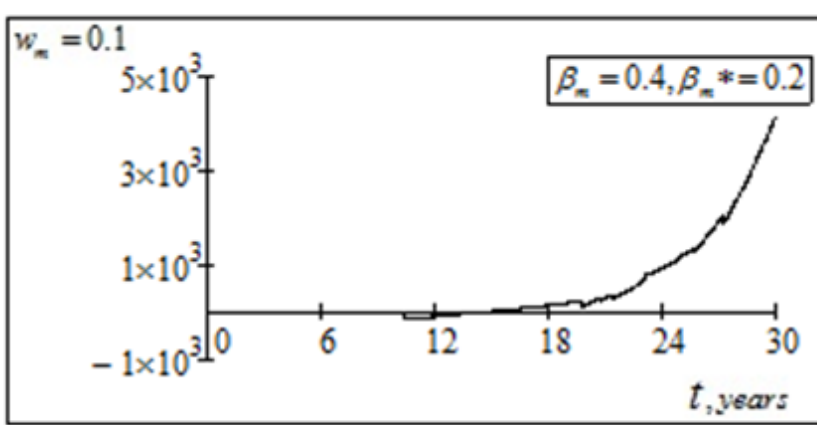

c). Graphical simulation of $w_{m}$ with predominant parameters, $\mathrm{r}=0.4, e, \mu, q, t_{\mathrm{w}}=0.5$

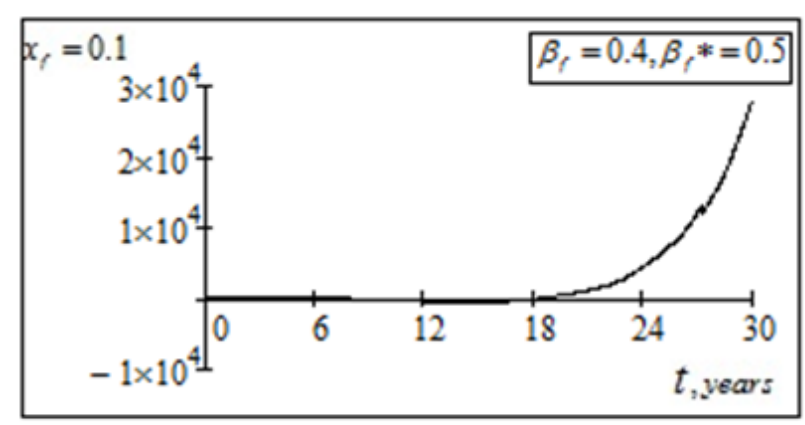

e). Graphical simulation of $x$, with predominant parameters, $\mathrm{r}=0.4, e, \mu, q, t_{\mathrm{w}}=0.5$

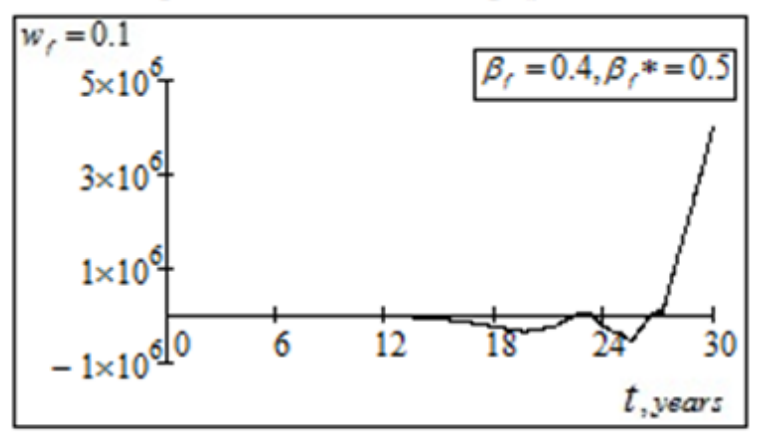

g). Graphical simulation of $w$, with predominant parameters, $\mathrm{r}=0.4, e, \mu, q, t_{\mathrm{w}}=0.5$ formative stage and through 27 years i.e. $u_{f} \leq 5 \times 10^{23}$. Female acquired intelligence is sharper at the later years i.e. for $t \geq 27$ years, $u_{f}=1.15 \times 10^{24}$.

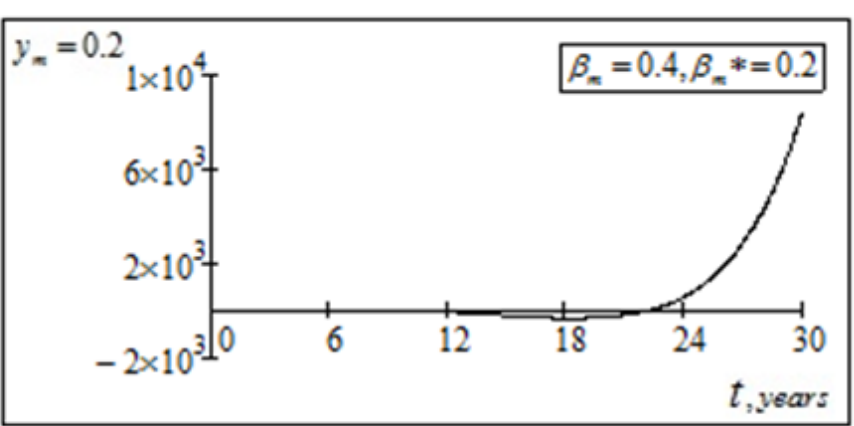

b). Graphical simulation of $y_{n}$ with predominant parameters, $\mathrm{r}=0.4, e, \mu, q, t_{\mathrm{w}}=0.5$

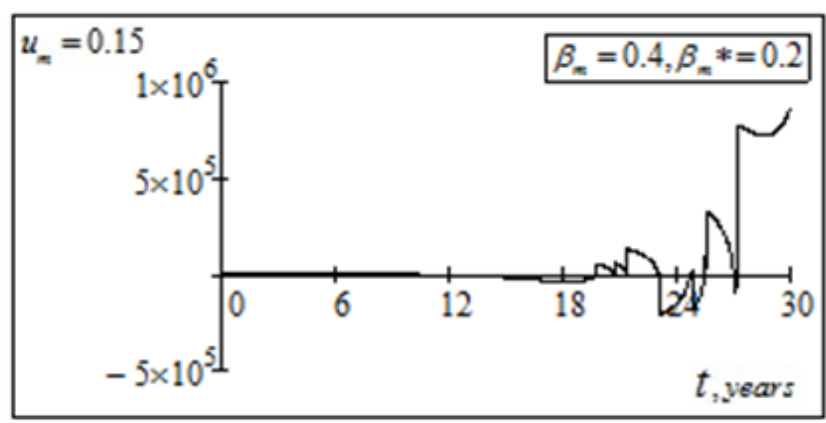

d). Graphical simulation of $u_{m}$ with predominant parameters, $\mathrm{r}=0.4, e, \mu, q, t_{\mathrm{w}}=0.5$

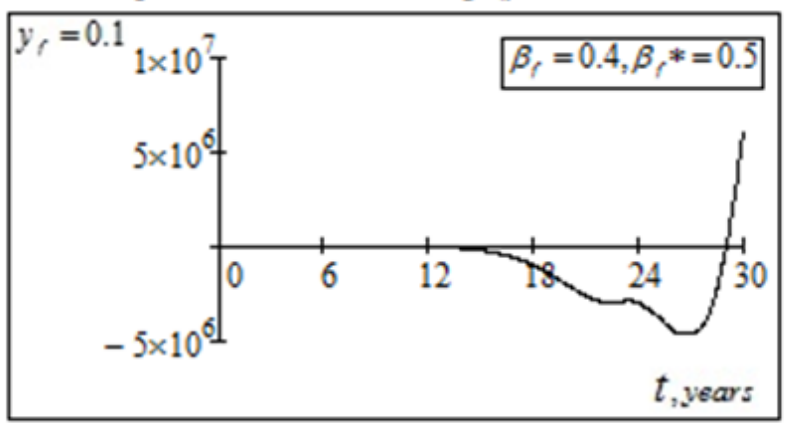

f). Graphical simulation of $y$, with predominant parameters, $\mathrm{r}=0.4, e, \mu, q, t_{\mathrm{w}}=0.5$

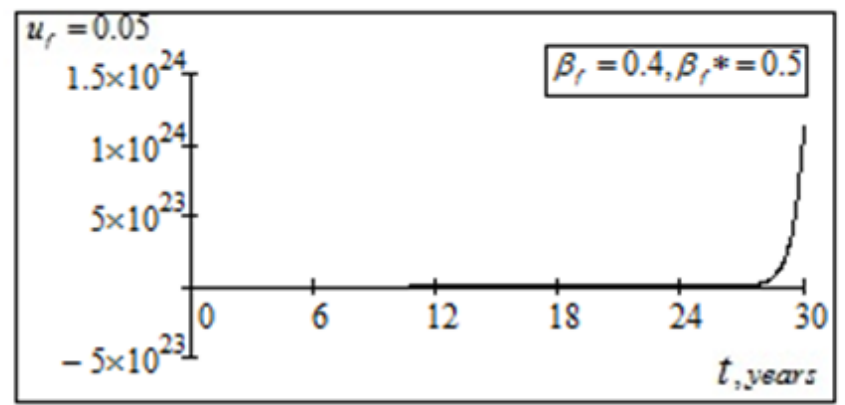

h). Graphical simulation of $u$, with predominant parameters, $\mathrm{r}=0.4, e, \mu, q, t_{\mathrm{s}}=0.5$

Figure 4 (a-h). Graphical simulation of male and female pupil/students' performance in mathematics with predominant parameters, $\mathrm{r}=0.4$, $e, \mu, q, t_{w}=0.5$ 


\subsection{Discussion}

In this paper, we had considered and implemented a mathematical model that accounted for 8-Dimensional mathematical differential equations for male and female performance in mathematics as a function of their parental background in the subject. The model explored quantitative ordinary differential equations (ODEs) and the analysis conducted using numerical computation. The model clinically analyses the varying intelligence levels of parental and students' performance in mathematics.

For simplicity, evaluation of the overall performance is considered in four major categories: parental knowledge of mathematics under varying studying environmental factors; males and females mathematical intelligence under zero availability of predominant parameters (i.e. $\left.e, \mu, q, r, t_{w}=0\right)$; male and female behavioral intelligence in mathematics under enhance predominant studying parameters (i.e. $r=0.4 ; \mu, q, \mathrm{t}_{w}=0.5, e=0$ ); and males and females behavioral intelligence in mathematics under enhanced predominant studying parameters intelligence (i.e. $r=0.4, e, \mu, q, t_{w}=0.5 \quad$, with $\beta_{m}, \beta_{f}=0.4$; $\beta_{m} *=0.2$ and $\beta_{f} *=0.5$.

The overall evaluation of parental background in mathematics indicated that for zero studying predominant parameters, the male parents are generally weak in mathematics with visible flash of knowledge at 7-10 years and having apex value $y_{m}=29.42 \%$. Performance of female parents was comparatively low at greater part, with $5.35 \%$ glimpse of mathematical knowledge at the $7^{\text {th }}$ year and complete deterioration to $-6.79 \%$ at the $10^{\text {th }}$ year. Under appreciable studying environmental predominant parameters, i.e. $\mu, q, t_{w}=0.5, r=0.4, e=0$, male parent population exhibited $2.11 \%$ sharpness in mathematics at the early stage with intermittent depreciation and then appreciated to $5.51 \%$ at the 27 through 30 years. Female parents exhibited some seeming lack of mathematical knowledge particularly at the early stage with inclined knowledge of $0.75 \times 10^{3} \%$ from the $27^{\text {th }}$ year. For a comprehensive provision of conducive environment with $e, \mu, q, t_{w}=0.5$ and $r=0.4$, analysis of result shows that male parents exhibited onset proficiency in mathematics with upward oscillation of intelligence from 12 years through 27 years. The level of male parents' hereditary and acquired intelligence advanced to a peak of $4.92 \times 10^{4} \%$ at the later stage of mathematical study. Comparatively, analysis of female parents' with hereditary and acquired intelligence exhibited slow initial assimilation of the subject. The proficiency of the female parents' was much more visible after 18 years through 30 years with apex value of $2.82 \times 10^{4} \%$. This latter analysis explains the fact that male parents' were twice brilliant in mathematics than their female counterparts under similar conditions.

Under zero predominant studying parameters $\left(e, \mu, q, r, t_{w}=0\right)$, male pupil/students with hereditary and acquired intelligence in mathematics exhibited flips of mathematical intelligence particularly at their formative stage and the interval of 12 years with value $8.65 \%$ and then diminished to as low as $-4.56 \%$. Female counterparts under similar conditions exhibited insignificant static knowledge of the subject with only $0.14 \%$ brightness as best level of intelligence. The male pupil/students with only hereditary intelligence but with zero predominant parameters exhibited a complete lack of mathematical knowledge. Here, intelligence for mathematics was worst at their $10^{\text {th }}$ year with value $-35.74 \%$ at formative stage. Their counterpart female students exhibited more spatial hereditary. Still under zero predominant parameters, male population under acquired intelligence showed prominence at formative stage in the $7^{\text {th }}$ year and were brighter at the $10^{\text {th }}$ year with value $u_{m}=9.27 \%$, which the depreciated thereafter. The female counterparts indicated marginal knowledge of the subject at the formative stage only to diminish completely after 10 years.

Analysis of intermediate availability of predominant studying parameters (i.e. $\mu, q, t_{w}=0.5, r=0.4, e=0$ ) for male pupil/students with combined hereditary and acquired intelligence showed that knowledge of mathematics was quite low at formative stage through $11^{\text {th }}$ year and then appreciated tremendously to a peak value of $y_{m}=48.71 \%$ from the $12^{\text {th }}-18^{\text {th }}$ year. Knowledge of the subject depreciated thereafter, a situation which can be attributed to lack of complete conducive environment, i.e. $e=0$. The female counterparts experienced docile knowledge of the subject at formative stage, which the improved significantly from the $18^{\text {th }}$ year to a value of $1.7 \times 10^{7} \%$. The implication here is that the females were more brilliant than their male counterparts from the $18^{\text {th }}$ year. Males under this condition with only hereditary intelligence revealed low understanding of the subject at formative stage but appreciated after 21 years with value of $w_{m}=4.12 \times 10^{4} \%$. Females' population with only hereditary intelligence that was subjected to intermediary predominant parameters exhibited gradual appreciation of mathematical intelligence at formative stage and then sprang with trapezoid inclination of value $2.99 \times 10^{6} \%$ after 17 years. We therefore observe here, that females with only hereditary intelligence were sharper compared to their male counterparts from 17 years. Under intermediate availability of predominant parameters, analysis of male pupil/students with only acquired intelligence experienced initial low knowledge of the subject at the formative stage. Knowledge of mathematics rejuvenated from $12^{\text {th }}$ year through $27^{\text {th }}$ years with peak value of $4.76 \times 10^{5} \%$. Females with only acquired intelligence under similar condition through showed slow knowledge of the subject but exhibited tremendous acquisition of mathematics only after $26^{\text {th }}$ year of study with apex value of $7.21 \times 10^{23} \%$. Thus, we inferred that the male were brighter compared to their female counterparts from $12-24$ years, while the females exhibited accelerated progress from $27^{\text {th }}$ year of knowledge acquisition.

Furthermore, under quantified comprehensive provision of studying predominant parameters i.e. $e, \mu, q, t_{w}=0.5$, $r=0.4$ together with enhanced intelligence transmission probability of $\beta_{f}^{*}=0.2$ and $\beta_{f}^{*}=0.5$, analysis showed 
that male pupil/students with combined hereditary and acquired intelligence in mathematics showed low and static apprehension of mathematics at formative stage through the $12^{\text {th }}$ year. Mathematical knowledge appreciated after 18 years and for the duration of investigation with peak value of $8.44 \times 10^{3} \%$. The female counterparts exhibited stationary growth of intelligence in the subject at formative stage through 13 years with undulating amplitude through $26^{\text {th }}$ year that has it peak $y_{f}=6.18 \times 10^{3} \%$. This result showed that females at 26 and above are more intelligence than male counterparts. To an apex value of $4.15 \times 10^{3} \%$, the male population with only hereditary intelligence in the presence of comprehensive studying environment exhibited positive evolvement of hereditary knowledge from 15 - 30 years of the investigation. The female counterparts possess basic knowledge of the subject at formative stage with $w_{f} \leq 1 \times 10^{6} \%$ and the diminished to $-5.09 \times 10^{5} \%$ in the interval $16 \leq w_{f} \leq 27$ years. After 27 years, knowledge of mathematics appreciated to $w_{f}=4.03 \times 10^{6} \%$. The implication here are that for both males and females, the understanding of the subject was more visible at their later years of studying the subject with that of the females sharper. Finally, the behavioral intelligence of both male and female pupil/students with only acquired intelligence in mathematics under enhance learning environment proved that males with acquired knowledge exhibited general low intelligence in mathematics at formative stage with zero value at 10-14 years. The male acquired intelligence exhibited gradual appreciated from 15-30 years of experimental study with apex value of $4.15 \times 10^{3} \%$. Their female counterparts were seen brighter compared with the males at the formative stage with $u_{f} \leq 5 \times 10^{23} \%$ through 27 years and even sharper at $t \geq 27$ years with $u_{f}=1.15 \times 10^{4} \%$. Thus, the overall results indicated that fever females appreciated the subject - mathematics and exhibited sharper intelligence compared to their male counterparts, under acquired knowledge in mathematics.

\section{Conclusion}

In this paper, ordinary differential equations were used for the formulation of an 8-Dimensional mathematical dynamic model for the study of pupil/students' behavioral intelligence in mathematics as a function of parental background. The method of analysis explored numerical quantitative approach, which classified resulting outcome into four categories: parental knowledge of the subject at varying accessibility of learning environment; pupil/students mathematical intelligence under zero availability of studying predominant parameters; pupil/students behavioral intelligence in mathematics under intermediary (semi-) availability of predominant parameters and pupil/students' performance in mathematics given enhanced predominant parameters.
From our computational analysis, its viewed that male parents with combined hereditary and acquired intelligence in mathematics were sharper and brighter compared with the female counterparts under enhanced accessibility of studying predominant parameters and was far worse when predominant parameters was completely zero. The result depicted the intelligence of pupil/students' performance in mathematics as the population with combined hereditary and acquired intelligence exhibited brilliant knowledge of the subject after 18 years with the females showing more better understanding as time dependent. Furthermore, result showed general low appreciation of the subject at formative stage by both male and female pupil/students and as well, revealed that under intermediary accessibility of predominant parameters, females with only hereditary intelligence were sharper from 18 years compared to their male counterparts whereas, the males with acquired intelligence were brighter than their female counterparts. The model therefore, suggest the inevitable need for more collaboration of parents, government and governmental agencies to foster more endurable and sustainable studying environment for the upgrade of pupil/students' intelligence in mathematics. Furthermore, a broader model with more improved predominant studying parameters is thus encouraged.

\section{References}

[1] Fafunwa, A. (1974). History of Education in Nigeria. London, George Allen and Unwin, pp. 22-31.

[2] Adeyemo, P. P. (1972). Principle and Practices of Education. OdoEkiti, pp. 11.

[3] Amadi, L. E. (1981). Principle of Curriculum Development Legacy, p. 12.

[4] Collin, G. (1902). English Dictionary, London, pp. 280.

[5] Hilgard, E., Richard, C., Alkinson, P. and Rita, H. (1933). Level of Aspiration as Affected by Relation Standing in an Experimental psychology, London, pp.13-27.

[6] Fatienla, S. O. (1985). Computational: proceeding of the first international conference in numerical analysis and its appreciation, ed., p. 2.

[7] Kreyszig, E. (1980). Advanced Engineering Mathematics. London, p. 940.

[8] Peter, H. (1973). Elements of Numerical Analysis. McGraw-Hill Incorporated p. 3.

[9] Anderson, R. M. and May, R. M. (1991). Infectious Diseases of Humans: Dynamics and Control, Oxford University Press, N. Y. pp. 290-292.

[10] Kimbir, A. R., Musa, S. and Bassey, E. B. On a two-sex Mathematical model for the prevention of HIV/AIDS in a varying population//J. Math. Assoc. Nig., 2006. 33, 201, pp 1 -13.

[11] Boaler, J. and Selling, S. K. (2017). Psychological imprisonment or intellectual freedom? a longitudinal study of contrasting school mathematics approaches and their impact on adults' lives. http://www.nctm.org/Publications/Journal-for-Research-inMathematics-Education/2017/Vol48/Issue1/PsychologicalImprisonment-or-Intellectual-Freedom_-A-Longitudinal-Study-ofContrasting-School-Mathematics-Approaches-and-TheirImpacton-Adults_-Lives/ . Accessed February 22, 2017.

[12] Velasco-Hernandez, J. X. and Hsieh, Y. H. (1994). Modeling the effect of treatment and behavioral change in HIV transmission dynamics. J. Math. Biol., 32, pp 233-349.

[13] Megan, N. and Craig, J. C. (2017). Mathematical Thinking and Learning through Robotics Play for Children with Critical Illness: The Case of Amelia.

http://www.nctm.org/Publications/Journal-for-Research-inMathematics-Education/2017/Vol48/Issue1/Mathematical- 
Thinking-and-Learning-Through-Robotics-Play-for-ChildrenWith-Critical-Illness_-The-Case-of-Amelia/.

Accessed February 22, 2017.

[14] Toerner, G. and Arzarello, F. (2017) Grading Mathematics Education Research Journals. http://www.mathematik.uni-dortmund.de/ erme/doc/EMS-ERMERanking_Journals_Project.pdf . Accessed February 22, 2017.

[15] Dawkins, P. (2017) How to Study Mathematics. http://tutorial.math.lamar.edu/pdf/How_To_Study_Math.pdf. Accessed February 22, 2017. 